\title{
Vers l'enfer (sur les proses dites «évangéliques»)La première prose dite «évangélique»
}

\section{Yann Frémy}

\section{(2) OpenEdition}

\section{Journals}

\section{Édition électronique}

URL : http://journals.openedition.org/studifrancesi/34078

DOI : 10.4000/studifrancesi.34078

ISSN : 2421-5856

Éditeur

Rosenberg \& Sellier

\section{Édition imprimée}

Date de publication : 1 novembre 2005

Pagination : 272-291

ISSN : 0039-2944

\section{Référence électronique}

Yann Frémy, «Vers l'enfer (sur les proses dites «évangéliques»)La première prose dite «évangélique» », Studi Francesi [En ligne], 146 (XLIX | II) | 2005, mis en ligne le 30 novembre 2015, consulté le 18 avril 2021. URL : http://journals.openedition.org/studifrancesi/34078 ; DOI : https://doi.org/10.4000/ studifrancesi.34078 


\section{Vers l'enfer \\ (sur les proses dites «évangéliques»)}

\section{La première prose dite «évangélique»}

La première des proses dites «évangéliques» n'est pas particulièrement attractive. À vrai dire, il ne s'y produit que peu de choses: c'est un texte, à tous points de vue, bloqué. Celui-ci est-il pour autant rébarbatif? «À Samarie [...]» est une prose qui traite du blocage, de l'enfermement, de quelque impossible échappée. Mais comment représenter et dénoncer ce blocage autrement que par un texte bloqué lui-même?

La première prose dite «évangélique» retient ce qu'elle donne, mais cette rétention est également une donation de sens qui concerne de près Une saison en enfer. L'envers de ce blocage est donc la dimension prospective de ce texte, dont la latence même recèle une masse d'informations.

Un monde fermé

Une critique sociale: les topiques ou la reproduction du Même

«À Samarie [...]» est un poème en prose traitant de la fermeture du monde, de l'impossibilité de le renouveler, que l'on soit prophète ou poète. Les Illuminations se présentent par ailleurs comme une tentative de réévaluation et de réinvention d'un monde que Rimbaud juge décevant, peu conforme à son désir, qui est d'énergie.

Dans la quatrième partie de Jeunesse, le locuteur, songeur, déclare: «Quant au monde, quand tu sortiras, que sera-t-il devenu? En tout cas, rien des apparences actuelles». Le prophète arrive dans la contrée de Samarie, dans la ville de Sychar probablement, mais rien ne peut s'y passer. Rimbaud adapte le texte évangélique dans le sens d'une aggravation des blocages; en effet, dans l'Évangile selon saint Jean, bien que le Christ soit chez un peuple hostile, sa parole, en dépit des résistances éprouvées, parvient à s'affirmer:

Et il y eut beaucoup davantage qui crurent en lui, pour l'avoir entendu parler.

De sorte qu'ils disaient à cette femme: Ce n'est plus sur ce que vous nous avez dit que nous croyons en lui; car nous l'avons ouï nous-mêmes, et nous savons qu'il est vraiment le Sauveur du monde ${ }^{1}$.

Un tel effet est manifestement absent des proses dites «évangéliques». Avant de conclure à une quelconque impuissance du Christ, il faut jauger le degré de résistance de cette province face au renouvellement que le Christ ne manque pas de lui proposer. D'une certaine manière, Antoine Adam a raison de voir dans cette série de proses une manière de critique sociale. Selon ce dernier,

[i]l saute aux yeux que Rimbaud pratique ici l'anachronisme systématique. Samarie, c'est l'Angleterre, la perfide Albion, le pays qui étale sa richesse, son égoïsme, et se targue d'observer sa loi protestante. Elle est le symbole de la société industrielle et capitaliste².

(1) Évangile selon saint Jean, IV, 41-42. La traduction prise en compte est celle de Lemaître de Sacy.
(2) Rimbaud, Euvres complètes, édition établie par Antoine Adam, Paris, Gallimard («Bibliothèque de la Pléiade»), 1972, p. 1024, n.1. 
L'identification de Samarie à l'Angleterre est fragile, mais la perspective adoptée est stimulante. Rimbaud superpose les lieux de sa vie récente à la contrée de Samarie, hormis le fait que, dans le cas présent, si nul n'est prophète en son pays, le poète l'est encore moins à l'époque de Rimbaud qu'à celle du Christ. La contrée de Samarie se présente comme un monde non seulement perfide (elle est «la parvenue, l'égoïste»), mais qui de surcroît semble se satisfaire de ses défauts et de ses faiblesses en les proclamant des vertus. Elle confère une forme officielle, institutionnelle, aux vices qui sont ceux des pires hommes, en les protégeant par des lois. Rimbaud décrit les mécanismes qui régulent la société de son temps, laquelle transforme les pires des vices en modèles de référence, en leur donnant un cadre, en leur conférant une légitimité sociale. De fait, ce que la contrée de Samarie adore, ce sont moins les «idoles» que sa propre immoralité dont, à bien des égards, elle se sent fière. Le dieu adoré est ici le dieu argent, et l'idolâtrie de Samarie, loin d'être païenne, n'a d'autre but que de tenir à distance cette autre religion qu'est le christianisme, si contraire aux valeursdéfauts dont se fait gloire cette contrée. L'envers d'une telle autosatisfaction dans la quotidienneté est la constitution d'un monde qui ne procède que par blocages, routines, codes:

Là la richesse universelle permettait bien peu de discussion éclairée. Le sophisme, esclave et soldat de la routine, y avait déjà, après les avoir flattés, égorgé plusieurs prophètes.

Non sans quelques ambiguïtés, le poème en prose des Illuminations intitulé Ville procède à une semblable condamnation des tendances fatalement égalisatrices de la civilisation occidentale, qui ne vont pas d'ailleurs sans quelques violences:

Je suis un éphémère et point trop mécontent citoyen d'une métropole crue moderne parce que tout goût connu a été éludé dans les ameublements et l'extérieur des maisons aussi bien que dans le plan de la ville. Ici vous ne signaleriez les traces d'aucun monument de superstition. La morale et la langue sont réduites à leur plus simple expression, enfin! Ces millions de gens qui n'ont pas besoin de se connaitre amènent si pareillement l'éducation, le métier et la vieillesse, que ce cours de vie doit être plusieurs fois moins long que ce qu' une statistique folle trouve pour les peuples du continent.

Rimbaud superpose donc l'épopée du Christ à des impressions de sa vie contemporaine. Le monde des villes - Sychar, Paris ou Londres - est fait de rétentions, $\mathrm{d}$ 'avarices, de blocages. Les codes règnent sur ce monde paranoïaque, qui n'accepte ni la contestation, ni le moindre changement. Rimbaud ne fait que rendre compte de la dictature des codes, de la prolifération des représentations et de l'impossibilité de ne pas s'y conformer. C'est l'époque du consensus, du grand murmure anonyme, de la fin (provisoire) de l'Histoire, dont Une saison en enfer propose une version différente, particulièrement ironique:

Qu'étais-je au siècle dernier: je ne me retrouve qu'aujourd'hui. Plus de vagabonds, plus de guerres vagues. La race inférieure a tout couvert - le peuple, comme on dit, la raison; la nation et la science.

Oh ! la science ! On a tout repris. Pour le corps et pour l'âme, - le viatique, - on a la médecine et la philosophie, - les remèdes de bonnes femmes et les chansons populaires arrangés. Et les divertissements des princes et les jeux qu'ils interdisaient! Géographie, cosmographie, mécanique, chimie !... il pas?

La science, la nouvelle noblesse! Le progrès. Le monde marche! Pourquoi ne tournerait-

C'est la vision des nombres. Nous allons à l'Esprit ! [...] 
Que faire dans un monde pareil, dans ce monde du Pareil? Davantage que contre l'artifice, Rimbaud lutte contre la facticité, c'est-à-dire qu'il s'insurge contre un type de société qui extrait un monde «partiel et partial» à partir du monde «total». La modernité, ce poison, érige un monde fait de codes et de reproductions des mêmes gestes, des mêmes paroles, à l'intérieur d'un monde pourtant total, ouvert, en expansion, qui est celui du possible; et Rimbaud a en horreur ces sociétés qui absolutisent des valeurs somme toute relatives et s'avèrent incapables de saisir le caractère arbitraire et inique de leurs pratiques (qu'un déluge, un jour, emportera). Le monde moderne est bloqué, ce qui ne va pas, Rimbaud le sait, sans une certaine violence, sans que ne soient présentes les pulsions les plus viles. Le poète a déjà indiqué que la contrée de Samarie, en allégorie de la modernité, avait transformé ses défauts en de paradoxales vertus. Le constat s'aggrave par la suite, puisque Rimbaud montre explicitement que les codes se nourrissent eux-mêmes de l'énergie révolutionnaire et que la paranoïa sécuritaire des villes est indissociable d'une part de schizophrénie, qu'elle absorbe puis qu'elle régule. Les sociétés modernes ne sont pas des mondes clos d'avance: ce sont des machines intensives à caractère transformationnel. Leur fonction est de changer l'énergie potentiellement révolutionnaire en codes parfaitement réactionnaires. Par exemple, le narrateur estime que «la richesse universelle permettait bien peu de discussion éclairée». La richesse universelle implique la présence d'un système économique ouvert, au sein duquel ont lieu des échanges, où existe une certaine circulation des biens et des personnes. La créativité humaine (à savoir les productions désirantes) connaît alors des déterminations sociales secondaires: elle est pernicieusement requalifiée. Ce potentiel créateur est donc dévoyé, placé sous un joug socio-économique totalement réactionnaire.

\section{Imposture transcendantale}

Au lieu de servir à une quête fiévreuse de la vérité, l'impulsion créatrice engendre des objets finis, sans ambition autre que de tendre à l'homme un miroir de satisfaction. Le voyant perçoit dans cet afflux de richesse, dans l'œuvre-miroir construite par la société samaritaine, un véritable danger: celui d'une stase sur soi dans l'opulence, un retour des «merveilleuses images» ${ }^{3}$ stériles qui permettent de voir la vie en beau. L'anti-héros du Mauvais Vitrier réclamait des verres pour voir «la vie en beau» ${ }^{4}$, mais Baudelaire refusait finalement une telle définition de la poésie. Rimbaud craint visiblement que la société marchande, en répandant ses produits, crée une sorte de leurre d'absolu qui rendrait inutiles poètes et prophètes. Face au peuple fasciné par les «merveilleuses images», quels peuvent être l'absolu et l'enseignement du poète et du prophète? Dans le poème intitulé Bonne pensée du Matin, Rimbaud s'inquiétait déjà de ce que les forces vives des Ouvriers puissent être utilisées à des fins ouvertes de facticité:

Dans leur désert de mousse, tranquilles,

Ils préparent les lambris précieux

Où la richesse de la ville

Rira sous de faux cieux (c'est moi qui souligne).

Rimbaud dénonce donc cette nouvelle transcendance du commerce qui pourrait bien un jour se substituer à la poésie.

(3) Cette expression figure dans Après le Déluge. En prenant acte de cette modernité monstrueuse, le poète la condamne certes moralement, mais il en tire profit poétiquement.
(4) Voir le poème en prose intitulé «Le Mauvais Vitrier», in Le Spleen de Paris, Euvres complètes, tome I, édition établie par Claude Pichois, Paris Gallimard («Bibliothèque de la Pléiade»), 1974, p. 287. 


\section{La violence des sociétés modernes}

La transcendance devient imposture. Mais Rimbaud a surtout soin de dénoncer la violence qui habite ce type de société: «Le sophisme, esclave et soldat de la routine, y avait déjà, après les avoir flattés, égorgé plusieurs prophètes». Cette société se présente comme ouverte à la nouveauté, assurément tolérante, prête en toutes circonstances à accueillir l'Autre («après les avoir flattés»), mais finalement se révèle un modèle d'intolérance et de totalitarisme larvé. Dans le poème en prose des Illuminations intitulé Démocratie, Rimbaud démasque de manière comparable la violence meurtrière qui sous-tend des systèmes sociétaux ne proposant en fait que des simulacres de liberté réelle. Codes, imposture transcendantale, violence meurtrière secrète qui menacent tout acteur du changement, sont donc les trois griefs que le poète adresse aux sociétés de type samaritain, derrière lesquelles l'on devine aisément que se dissimulent «ces Paris-Londres moins que laids ${ }^{5}$.

\section{Un langage usé}

À ce monde factice correspond une parole dont le cours suit nécessairement celui de «l'universel reportage» honni par Mallarmé ${ }^{6}$. Le manque d'énergie de la langue caractérise, selon Rimbaud, les sociétés «crue[s] moderne[s]» et assure une certaine continuité des rapports sociaux amorphes au langage en charge de les exprimer. Nulle rupture ne vient donc remettre en cause cette pernicieuse alliance: le monde n'exprime plus le possible, de même que l'énergie déserte le langage. Langage et Monde sont placés sous l'égide du Même et valident des représentations identiques: les mots sont désormais à l'image des choses. La facticité du monde s'exprime à travers le caractère anénergétique de la langue, ceci sans nulle anfractuosité: les sociétés de type samaritain inaugurent une nouvelle métaphysique, à laquelle un mélange de «déjà-vu» et de «déjà-dit» tient lieu de définition. Cette intrication du visible et de l'énonçable ne fait que célébrer l'inconsistance de toute existence. Dans la première prose dite «évangélique», Rimbaud dénonce donc l'inanité de toute parole. Paradoxalement, il utilise l'Evangile selon saint Jean pour dénoncer la vacuité moderne de la parole et son caractère stéréotypé, alors que Jean est avant tout l'apologiste de la «parole vive» du Christ. Selon l'apôtre, le Christ représente surtout le Verbe incarné, la Parole étant le principal moyen de conversion reconnu dans cet Évangile. L'Évangile selon saint Jean débute par une évocation de la Parole:

Au commencement était le Verbe, et le Verbe était avec Dieu, et le Verbe était Dieu

\section{Le Verbe habite ensuite le corps du Christ:}

Et le Verbe a été fait chair, et il a habité parmi nous; et nous avons vu sa gloire, comme la gloire du Fils unique du Père, étant plein de grâce et de vérité ${ }^{8}$.

L'épisode de la rencontre du Christ avec la femme samaritaine illustre bien le potentiel considérable de conversion propre à la parole christique. La femme reconnaît en Jésus un prophète et celui-ci s'annonce comme le Sauveur du monde. L'Évangile selon saint Jean contient d'autres détails:

(5) Verlaine, «À Arthur Rimbaud. Sur un croquis de lui par sa sœur», in Dédicaces, Euvres poétiques complètes, édition établie par Jacques Borel, Paris, Gallimard («Bibliothèque de la Pléiade»), 1972, p. 602.

(6) «Avant-dire au Traité du Verbe de René
Ghil», in Euvres complètes, édition établie et annotée par Henri Mondor et G. Jean-Aubry, Gallimard («Bibliothèque de la Pléiade»), 1945, p. 857.

(7) Evangile selon saint Jean, I, 1.

(8) Ibid., I, 14. 
Or il y eut beaucoup de Samaritains de cette ville-là qui crurent en lui sur le rapport de cette femme, qui les assurait: Qu'il lui avait dit tout ce qu'elle avait jamais fait.

Les Samaritains, étant donc venus le trouver, le prièrent de demeurer chez eux, et il demeura deux jours.

Et il y eut beaucoup davantage qui crurent en lui, pour l'avoir entendu parler.

De sorte qu'ils disaient à cette femme: Ce n'est plus sur ce que vous nous avez dit que nous croyons en lui; car nous l'avons ouï nous-mêmes, et nous savons qu'il est vraiment le Sauveur du monde?

La puissance dévolue à la Parole est donc considérable. La première prose dite «évangélique» modifie radicalement le texte johannique sur ce point. En effet, l'Évangile selon saint Jean est la relation de la parole vive, quand la libre adaptation qu'en propose Rimbaud est la consécration paradoxale de la parole dévaluée, dont le théâtre social ne consiste qu'en sophismes, en discussions peu éclairées, alors que le Christ fait silence. Le poète ne fait qu'indiquer un changement d'époque, une logique de durcissement. Le voyant tente d'imposer une neuve parole en vue d'un changement de monde en cette fin de XIX ${ }^{e}$ siècle, à l'instar du Christ il y a deux mille ans. Le Christ et Rimbaud souhaitent tous deux renouveler le monde à l'aide d'une parole rendue vivante, intensifiée. Or ce qui était déjà presque impossible à l'époque du Christ l'est encore davantage à celle de Rimbaud, laquelle semble avoir considérablement amélioré son imperméabilité à toute forme de changement. Comme Jean-Luc Steinmetz le souligne, Rimbaud ressent sa propre vie en celle de Jésus ${ }^{10}$, en dépit du fait que la tâche est aujourd'hui plus rude pour le voyant qu'elle ne l'était jadis pour le Christ. L'inanité de parole dont faisaient déjà montre les Samaritains est comme aggravée par les Samaritains de l'époque moderne. Rimbaud n'a plus qu'à constater que le monde de la parole est désormais mort, qu'il est un ensemble de signes vides de sens. Par ailleurs, le poète n'est pas le seul à évoquer cette facticité historique de la parole. À son tour, Villiers de l'Isle-Adam intéressera sa poétique à un tel problème, laissant des pages dont les échos sont fort proches des lamentations statiques d'Une saison en enfer ${ }^{11}$. Selon l'hypothèse défendue dans cette étude, Rimbaud veut infuser de l'énergie dans la langue, ce qui n'implique nullement de créer une nouvelle langue, mais de faire entrer la langue standard dans un devenir. Faire entrer la langue commune dans un devenir qui la minore, qui soit intensif, implique un bouleversement du monde à court terme, selon le Christ et le poète. Délibérément, Rimbaud étudiera le cas de figure de la parole multiple dans la troisième prose dite «évangélique» et, évidemment, dans Délires II-Alchimie du Verbe. Ici, nulle création, mais seulement l'affligeant constat d'une parole dévaluée, d'une fausse monnaie. Est-ce à dire que la parole dont rêve Rimbaud est à l'identique de la parole vive du Christ? Non: le poète ne fait que constater que de semblables résistances, aggravées encore à son époque, viennent grever tout exercice de parole nouvelle. Rimbaud ne s'intéresse pas en archéologue à cette parole qui se fige. Les méfaits en sont connus, puisqu'elle sous-tend et soutient une violence parfois meurtrière (notons que c'est le sophisme qui égorge les prophètes). Le langage propre aux sociétés modernes se caractérise donc par son manque d'énergie.

L'économie propre à la première prose dite «évangélique» recense plusieurs rapports possibles à la parole, au nombre de quatre précisément:

(9) Ibid., IV, 39-42.

(10) «Assurément, c'est moins le christ fondateur, référé au Père, qui $[\ldots]$ retient [Rimbaud] que l'être hors du commun «changeant la vie» et même la restituant» («Les proses évangéliques», in Le Champ d'écoute, Neuchâtel, À la Baconnière («Langages»), 1988, p. 131).

(11) A ce propos, voir BERTRAND ViberT, Villiers l'inquiéteur, Toulouse, Presses Universitaires du Mirail («cribles»), 1995. 
- Le mot sinistre: «Là, la richesse universelle permettait bien peu de discussion éclairée».

Rimbaud établit un rapport de proportion inversé entre le degré de richesse et la puissance de renouvellement de la parole. Cette phrase s'oppose à telle autre extraite de Mauvais Sang, où le poète prétend sans ironie que «[1] a richesse a toujours été bien public». Pour Rimbaud, la parole manifeste un désir d'absolu. Or la richesse universelle constitue pour l'homme une sorte de substitut du désir, délivre un satisfecit qui prend la place du désir. C'est la stratégie traditionnelle des sociétés dites «modernes»: faire prendre un objet socialement ou économiquement déterminé comme objet de désir pour l'objet $d u$ désir. La prolifération d'une telle richesse, d'un tel absolu, dont la mise sous conditions commerciales en fait un second absolu, un simulacre d'absolu, annule le véritable désir de richesse qui est celui du monde de la parole. Un satisfecit économique et matériel ayant été délivré, les hommes se contentent de se mirer au miroir de leur propre opulence et oublient de fait leur premier désir, véritable et authentique, qui était celui d'un monde rendu neuf grâce à une parole neuve. La tactique socio-économique passe donc par la réappropriation de l'idée d'absolu, par la mise en place d'un second absolu, d'un absolu second socialement déterminé qui se substitue à l'absolu premier, proprement révolutionnaire (ou cru tel). L'afflux de l'absolu de richesse annule donc le désir d'une parole authentique. Le désir d'absolu bassement satisfait sert donc une parole dévaluée: l'opulence matérielle en échange de l'abandon des rêves. L'énergie première propre au désir a donc été codée, codifiée, surdéterminée, de manière à produire des agencements réactionnaires de parole(s) morte(s), inoffensive(s).

- La parole sophistique: «le sophisme, esclave et soldat de la routine, y avait déjà, après les avoir flattés, égorgé plusieurs prophètes».

Qu'est-ce donc qu'une parole sophistique? La parole usée entretient des rapports curieux avec les tenants de la «parole vive» (les prophètes). La parole dévaluée ne saurait être reconnue comme telle: elle se veut un lieu de vérité, l'exact reflet des choses. Elle réclame pour elle un mentir-vrai. Aussi est-elle sophistique en ce sens qu'elle pourrait dépérir si étaient révélées au grand jour son inanité et son inconsistance. Elle tient donc à passer pour vraie car elle cautionne des représentations sociales réactionnaires, une fausse réalité dont le mensonge ne peut survivre qu'à la condition de ne pas être dévoilé comme mensonge. Quiconque soupçonnerait l'imposture mettrait en effet en péril la facticité d'un tel langage-monde. Cette parole fausse qui se veut vraie, Rimbaud l'appelle sophisme. Un sophisme est un:

A. Argument valide en apparence, mais en réalité non concluant, qu'on avance pour faire illusion aux autres, ou dont on se paie soi-même sous l'influence de l'amour propre, de l'intérêt ou de la passion.

B. Argument qui, partant de prémisses vraies, ou jugées telles, aboutit à une conclusion inadmissible, et qui ne peut tromper personne, mais qui semble conforme aux règles formelles du raisonnement, et qu'on ne sait comment réfuter ${ }^{12}$.

Rimbaud débusque la véritable nature de la parole sociale, qui fausse la réalité sensible, en propose une sorte de double fantomatique qu'elle fait ensuite passer pour la réalité même. Conformément à ce que l'on entend par «sophisme», la parole sophistique présente comme une valeur universelle ce qui n'est en fait qu'une affaire de pulsions, de petites jouissances destinées à préserver l'ordre social. Aussi la parole

(12) Article «Sophisme», in André LaLAnde, Vocabulaire technique et critique de la philosophie, volume 2, Presses Universitaires de France («Quadridge»), 1997 [1926], p. 1010. 
sophistique est-elle à la fois «esclave et soldat de la routine», c'est-à-dire parole que la routine accapare, sous dépendance, qui exprime le vide du monde, mais qui se révèle être également le corps conducteur de cette même routine dont elle constitue pourtant la première occurrence. L'exprimé sert le futur exprimé, cautionnant ainsi un circuit de production fermé de la parole. La «parole vive» des prophètes menace donc le petit règne borgne et matériel de la «parole sophistique». Le plus dangereux pour les tenants de la parole sophistique serait de désigner la parole vive des prophètes comme l'ennemie à abattre. Les usagers de ce type de parole pourraient en effet se croire léser d'une vérité importante et se prendre ainsi à l'autre parole. La relation de sympathie, voire de complaisance esquissée alors («après les avoir flattés »), constitue donc, aux yeux du monde, un spectacle de tolérance, d'invitation à l'échange. C'est également dans ce cas une manière pour les tenants de la parole «sinistre» de désacraliser la parole des prophètes, puisque l'accueil qui leur est fait semble indiquer l'absence de danger que comporte pour la parole sophistique l'énergie de la parole prophétique. Mais ce n'est dans ce cas qu'un trompe-l'œil, et les sociétés crues modernes, sous couvert d'une mise en scène de la tolérance et de l'acceptation d'autrui, ne tardent pas à révéler leur visage de haine: les prophètes sont égorgés. Je ne puis dire si, dans cette prose, la parole prophétique échoue, mais toujours est-il que sa simple présence permet de révéler la violence d'une société profondément attachée à l'usage factice de sa parole. Pour Rimbaud, la parole prophétique sert de révélateur, de miroir grossissant. En effet, Rimbaud ne croit guère à l'absolu d'une prophétie de la parole; il cherche d'abord à défaire la parole usée. Toujours est-il que l'on veut faire vivre les hommes dans le monde faux de la parole fausse.

- La parole sinistre: «C'était un mot sinistre, celui de la femme à la fontaine: "Vous êtes prophète vous savez ce que j'ai fait" ».

Le mot de la Samaritaine n'est pas sinistre uniquement parce qu'il est dangereux pour le Christ, mais pour une autre raison qu'expose Paola Ricciulli:

Le mot de la femme est qualifié de «sinistre». La femme, suivant le texte de saint Jean, n'a pas d'intellect. L'Évangéliste dit qu'elle n'a pas de mari, elle ne peut donc comprendre la parole de Jésus, sinon dans un sens tout à fait matériel. Voilà pourquoi le mot de la Samaritaine est «sinistre»: il s'agit d'une parole «morte », «usée», de laquelle rien ne peut naître, s'opposant donc à la vie, à la parole «vive» de Jésus ${ }^{13}$.

La Samaritaine est une adepte de la parole sociale et elle répond à Jésus comme à l'un des potentats de la Cité, comme si elle avait quelque chose à se reprocher: «Vous êtes prophète, vous savez ce que j'ai fait». Effectivement, s'il y a quelque chose de sinistre, c'est bel et bien dans l'incapacité de la femme à saisir le caractère nouveau, révolutionnaire, de la parole christique. Dans l'Évangile selon saint Jean, le Christ répond à la Samaritaine:

[...] Quiconque boit de cette eau aura encore soif, au lieu que celui qui boira l'eau que je lui donnerai, n'aura jamais soif;

Mais l'eau que je lui donnerai deviendra dans lui une fontaine d'eau qui rejaillira jusque dans la vie éternelle $e^{14}$.

«[L'] eau que je lui donnerai»: de fait, la Parole vive. Il est vrai que dans cet Évangile la femme samaritaine reste peu sensible à la parole vive et l'est davantage

(13) «Rimbaud et la poésie de l'inexprimable dans la suite johannique», in Rimbaud cent ans après,

Parade sauvage, colloque $\mathrm{n}^{\circ} 3,1992$, p. 110-111.

(14) Evangile selon saint Jean, IV, 13-14. 
à la prophétie. Rimbaud aggrave ce donné: la femme samaritaine est en proie à une parole fausse, si bien que la parole vive n'a aucun impact sur elle. Rimbaud écrit son époque. Et l'on comprend mieux que Jésus refuse de voir ceux qui «ont manifesté leur foi en lui», puisque ceux-ci l'attendent probablement pour l'égorger dans ce monde de la parole de mort. Pire, il risque lui-même d'y perdre la parole, car l'on peut interpréter cette phrase dans un sens métaphorique:

Le sophisme, esclave et soldat de la routine, y avait déjà, après les avoir flattés, égorgé plusieurs prophètes.

Que le sophisme égorge les prophètes ne signifie pas obligatoirement qu'ils sont assassinés, mais que la parole dévaluée éteint la parole vive, lui ôte son énergie au point de la transformer en une parole morte. Rimbaud évoque le risque qu'encourent la parole du prophète et le prophète lui-même. Le langage de ce dernier risque d'être corrompu, le révolutionnaire rentrant alors dans le rang et acceptant l'usage social et sophistique de la parole, quitte à égorger à son tour les prophètes nouveaux qui se présenteront à lui. À Samarie, le monde du langage est totalement bloqué, dangereux même pour celui qui défend une conception énergétique de la langue. Risque d'entropie, de récupération. Le poète se sert assurément du texte évangélique pour évoquer la tragédie de la parole propre à son époque.

- La parole absente: «Jésus n'a rien pu dire à Samarie».

$\mathrm{Si}$ «Jésus n'a rien pu dire à Samarie», c'est en raison du fait qu'il était «incapable de la menacer matériellement». Qu'est-ce à dire, sinon que, face à la parole dévaluée, la parole prophétique s'avère inopérante $?^{15}$ Il s'agit d'un rapport de forces, entre la force «réactive» de la parole sinistre et la force «active» de la parole nouvelle. Mais, dans un certain sens, le combat n'a pas lieu, la lutte étant trop inégale. La capacité de résistance de la parole «sinistre» est telle qu'elle ne saurait être entamée par la parole prophétique. Nulle percée ne vient menacer ce qui peut apparaître à Rimbaud comme l'énergie propre à l'inertie. Faire bouger le monde (de la parole) est chose impossible. Comment mettre le feu (de la prophétie) à la langue? Comment entraîner une langue bloquée dans un devenir-intensif? Le personnage du voyant perce derrière celui du Christ, mais ce voyant est désabusé: il a buté contre le mur de la parole morte, en dépit de quelques vagues «succès d'estime» (que relate de manière cryptée, à mon sens, cette phrase où est livrée l'impression laissée par le poète à son nouvel entourage lors de son arrivée à Paris en septembre 1871: «À Samarie [Samarie désignant Paris], plusieurs ont manifesté leur foi en lui. Il ne les a pas vus »). Le monde ne sera pas changé. À l'heure de la Saison, Rimbaud en est conscient: une différence existe entre son désir de changement et le monde réel involutif. Ainsi la première prose dite «évangélique» sonne-t-elle le glas des assauts du voyant quant à la parole, dans son désir, du moins, de bouleverser les mœurs d'une époque en révolutionnant la langue. La prose aboutit moins au silence qu'à une retenue, presque une pudeur du Christ face à ce qui ne serait qu'une parole inefficace. La parole du Christ-voyant n'a peut-être pas perdu les hommes, mais elle ne les a pas non plus sauvés. Elle est ce qui n'agit pas: elle est également une parole morte, qui n'a plus rien à dire et ne peut plus se dire.

(15) Dans l'Évangile selon saint Luc (IX, 52-54), les disciples proposent à Jésus de faire descendre le feu du ciel pour dévorer les habitants d'un village de Samarie qui n'ont pas voulu le recevoir.
Toutefois, le Christ refuse, non pas en raison d'une quelconque incapacité matérielle, mais parce que, répond-il, il n'est pas ici-bas «pour perdre les hommes, mais pour les sauver» (IX, 56). 


\section{La deuxième prose «évangélique»}

La puissance de la nature

La deuxième prose dite «évangélique» débute par une notation des plus étranges: «L'air léger et charmant de la Galilée». Peut-être s'agit-il, comme on l'a soutenu avec vraisemblance, d'une allusion à La Vie de Jésus d'Ernest Renan ${ }^{16}$, mais là n'est pas l'essentiel. Entre le début et la fin du texte, une continuité existe sous la forme d'un chant de gloire consacré à la puissance de la nature. Toujours, Rimbaud a été réceptif aux forces qui agitent la nature: entre le poète néo-païen de Credo in unam... et celui qui raille la Mère Nature dans la lettre de mai 1873, il n'y a guère de contradiction. Que le poète adule la nature ou, au contraire, l'exècre, sa capacité à la jauger, à la goûter, à la ressentir en profondeur, demeure la même. Rimbaud est un consommateur de cette nature qu'il explore de fond en comble, dont il s'empare et capture les forces. Suite à cette consommation, plusieurs réactions sont possibles, de l'extase à la phobie. Dans la deuxième prose dite «évangélique», Rimbaud s'attache donc à décrire l'atmosphère propre à la Galilée: il tire de cette matérialité une idée. «L'air» est qualifié de «léger», ce qui est une notation physique, presque météorologique. Dans un premier temps, le nouvel évangéliste transcrit un certain état des choses, procède à une saisie objective du réel: la poésie rimbaldienne est matérialiste. Dans un second temps, l'air est qualifié de «charmant», la description tirant vers une plus grande subjectivité: l'idée naît de la matière. Un zeugme de construction prend donc place au linteau de la deuxième prose dite «évangélique». Rimbaud se montre sensible à la qualité de la réalité qui lui est proposée, à sa consistance, avant même d'évoquer la figure du Christ, laquelle est non seulement d'importance secondaire, mais est elle-même immergée au cœur de cette nature, avec laquelle elle entretient des rapports problématiques. Rimbaud se montre extrêmement sensible aux variations d'atmosphère, consomme les intensités naturantes qui constituent le biotope des hommes, qui sont même premières par rapport à eux. Elles sont également premières par rapport à ce Christ qui vient après la Création. Rimbaud est le poète physicien qui refuse de croire que le grand Pan est mort; il est celui qui refuse de se convertir à l'autre Dieu qui a attelé les hommes sur sa croix. Même si le goût de Rimbaud pour la nature semble s'être affiné par rapport aux soifs et aux faims du cycle de 1872 et aux cascades lyriques de Credo in unam..., le poète demeure néo-païen, au sens le plus exact du terme, comme l'a montré Laurence Watson ${ }^{17}$. La fin du texte reprend et prolonge l'évocation initiale de l'énergie de la nature. Il ne s'agit plus désormais d'une discrète évocation qualitative, mais d'une véritable explosion de puissance, d'une démonstration des forces de la nature. Les miracles du Christ se trouvent assurément en concurrence avec cette mise en scène de la nature par elle-même. Dans l'incipit, la nature n'était encore qu'une puissance en attente, qu'une latence, qu'un chaos potentiel, alors qu'elle se déclare totalement, à son maximum, au cours du «finale» que nous offre Rimbaud. Au comble de sa puissance, le réel est plus que le réel: la nature est en proie alors à une sorte de matérialisme «magique». Dès lors, la nature est dotée d'une énergie formidable, d'une puissance d'illumination qui la rend à sa gloire première. La nature, intense, ardente, compose avec d'autres puissances. Dans Après le Déluge, les «pierres précieuses» conquièrent le pouvoir de se cacher, tandis que les fleurs peuvent voir: «Oh les pierres précieuses qui se cachaient, - les fleurs qui regardaient déjà». Dans la première partie d'Enfance, de manière plus significa-

(16) Voir Yves Reboul, De Renan et des proses évangéliques, Parade sauvage, $\mathrm{n}^{\circ} 11$, décembre 1994, pp. 88-102.
(17) Notamment dans l'article intitulé «Rimbaud et le Parnasse», in Rimbaud ou «la liberté libre», Parade sauvage, colloque $\mathrm{n}^{\circ} 1,1987$, p. 18-29. 
tive encore, «les fleurs de rêve tintent, éclatent, éclairent». Dans la deuxième partie du même poème, elles «bourdonn[ent]». La puissance de la nature est portée à un tel degré d'intensité que celle-ci entre dans des relations non formelles et purement intensives avec d'autres potentiels. «[L]iserons» et «bourraches» sont des plantes d'une espèce commune et fort répandue: voici, néanmoins, que celles-ci rayonnent d'une vie proprement inconnue et qu'une énergie insoupçonnée voit le jour, comme si c'était le résultat de quelque rituel magique, comme si le «commun», ou supposé tel, avait eu droit à sa «minute d'éveil», comme si l'ingrat avait répondu par l'affirmative aux «virtualités de gloire de l'homme ${ }^{18}$. L'énergie embellit la nature la plus revêche, apparemment la plus rétive au changement.

Chaque chose s'ouvre en son milieu pour accoucher de sa potentialité magique, pour livrer son trésor. Les plantes magiques dont Rimbaud fait mention sont tout à fait remarquables: l'accent est mis sur leur lueur, leur incandescence, leur lumière, signe que leur énergie est pleinement active. La lueur magique n'est que la partie visible de l'intensité portée à son paroxysme. Celle-ci scintille «entre les pavés»: la précision est d'importance. En dehors du fait qu'elle renvoie à une traditionnelle opposition entre «nature» et» culture», elle indique un retour victorieux de la nature par rapport aux réalisations humaines (même dans les plus architecturales des Illuminations, Rimbaud ne fait qu'accompagner l'énergie de la nature: il ne s'y substitue pas). Le monde figé de la première prose dite «évangélique» s'anime, conquiert des puissances de vie et, fussent-elles encore cachées ou minoritaires, elles n'en sont pas moins réelles.

«Enfin il vit au loin la prairie poussiéreuse, et les boutons d'or et les marguerites demandant grâce au jour»: le paysage qu'observe Jésus est d'une grande incandescence. Son regard embrasse l'horizon («la prairie poussiéreuse»), avant de s'attacher aux existences particulières («les boutons d'or», «les marguerites»). En une phrase, Rimbaud reconstitue un monde total. La participiale «demandant grâce au jour» a pu surprendre: elle étonne moins si l'on se souvient que Rimbaud est un créateur de mondes, un démiurge. Le poète recrée un monde un, où l'énergie est la mesure de chaque chose, où d'infinis prêtés-rendus ont lieu entre microcosme et macrocosme. C'est le monde conçu comme gigantesque machine énergétique, quand les fleurs parlent au jour et que le jour les inonde de lumière. Il est vrai que la notation finale agit comme un signe à deux lectures possibles: la constitution d'un monde d'échanges n'interdit pas une autre interprétation, complémentaire, que défend Suzanne Bernard, selon laquelle les fleurs implorent la miséricorde du jour. Suzanne Bernard a noté que les fleurs demandaient leur grâce au jour à cause de la chaleur environnante ${ }^{19}$. Cette interprétation traduit la situation d'exposition extrême qui est celle du sujet face au feu qui règne sur le monde. Êtres de qualité, plutôt que simples réceptacles des intensités, les «boutons d'or» et les «marguerites» ne peuvent résister à la pression du jour et à la dépression de l'énergie. L'excessive énergie met en danger l'intégrité qualitative et figurative du sujet: la fin de la deuxième prose dite «évangélique» préfigure à sa manière Délires IIAlchimie du Verbe, où le poète-locuteur, soumis au feu roulant des intensités, apparaît, en maints endroits, calciné. S'agit-il, dans le cas présent, d'une sorte de portrait floral de Rimbaud?

L'insoutenable légèreté de l'être

La Galilée est très différente de Samarie, une province que Rimbaud ne portera jamais dans son cœur: dans Métropolitain, le poète raille «les derniers potagers de

(18) Yves Bonnefoy, Rimbaud, Paris, Éditions du Seuil («Écrivains d'aujourd'hui»), 1979 [1961], p. 150 .
(19) Rimbaud, Euvres, édition établie par Suzanne Bernard et André Guyaux, Paris, Garnier, 1991, p. 455. 
Samarie». Le peuple galiléen diffère fortement du peuple samaritain, et porte en lui les germes de ce peuple futur rêvé par Rimbaud. Ce peuple n'attend pas d'être sauvé car il semble déjà s'être sauvé lui-même. Peut-être a-t-il retrouvé sa propre jeunesse. Le monde bloqué, lourd et sans gloire de Samarie, laisse place à celui, léger, de la Galilée. Rimbaud considère ce peuple nouveau comme porteur d'une «joie curieuse», ne s'embarrassant que de peu de considérations morales, suffisamment joyeux pour ne pas être influencé par la morale essentiellement négative et profondément triste dont est porteur le Christ. Ce peuple non mélancolique n'attend pas le Christ: de fait, il prend la «sainte colère» du Christ pour un simple «miracle de la jeunesse pâleet furieuse». La seule énergie à laquelle il croit est humaine, immanente. Il ramène donc l'action du Christ à ses propres valeurs, sans qu'il s'agisse, comme dans la première prose dite «évangélique», d'un réductionnisme assassin, car dans le cas présent le peuple galiléen a su créer ses propres valeurs en communion avec les forces de la nature. Ce peuple nouveau est celui dont Rimbaud parlera dans Matin. La subtilité du texte vient de ce que Rimbaud attribue à un monde pré-chrétien (le Christ arrive à peine) les valeurs post-chrétiennes qu'il défend (dont il aimerait qu'elles soient pleinement les siennes: c'est tout le problème d'Une saison en enfer). Dans ce cas, il s'agit d'un véritable dépassement du christianisme, d'un retour des forces vives de l'être humain par delà la tristesse inhérente à la religion chrétienne. À cet effet, Rimbaud est très proche de Nietzsche dans L'Antéchrist:

Le christianisme a pris le parti de tout ce qui est bas, vil, manqué, il a fait un idéal de l'opposition, à l'instinct de conservation de la vie forte ${ }^{20}$.

On ne dit pas «néant»: à la place, on dit «au-delà», ou «Dieu», ou «vraie vie», ou bien «nirvana», «rédemption», «béatitude»... Cette innocente rhétorique née de l'idiosyncrasie religieuse et morale apparaît beaucoup moins innocente dès que l'on comprend quelle tendance se drape ici dans le manteau des grands mots: c'est celle de l'bostilité à la viee .

Dans le christianisme, ni la morale, ni la religion n'a aucun point de contact avec la réalitée ${ }^{22}$.

Le problème de Nietzsche est celui de Rimbaud: tous deux tentent de faire revenir la vie, de reconquérir cette énergie que le christianisme a volée, comme l'attestent Les Premières Communions. Dans la ville, certes, l'on trouve des «bourgeois» ou encore cet officier royal «aux mains chargées de bagues»: la richesse de la ville rit toujours sous de faux cieux; ce n'est pas exactement le peuple que Rimbaud rêve de réinventer, mais ce peuple donne l'impression ineffable d'une légèreté de l'être véritablement post-chrétienne dont on ne peut faire l'économie. Des flux d'énergie joyeuse parcourent la Cité: la tête de l'officier royal est «assez plaisante, quoique à demi chauve»; suite au «miracle», celui-ci s'en ira «comme on porte quelque pharmacie légère». Ce qui est marquant n'est pas la désinvolture descriptive de Rimbaud, mais, dans le bon sens de l'expression, le manque de sérieux du peuple galiléen. Le pathos chrétien ne l'atteint pas, ni l'orgueil, et il retrouve le sens du jour, celui de la légèreté de l'être, comme si le christianisme n'avait pas de prise sur lui. La force de ce peuple est de conserver l'énergie qui est la sienne, sans céder aux propositions ascétiques, à la gloire de tristesse et au sens du tragique du christianisme. Celui-ci est sans impact sur ce peuple qui a choisi de faire de l'affirmation de la vie son but suprême:

Jésus n'avait point encor fait de miracles. Il avait, dans une noce, dans une salle à manger verte et rose, parlé un peu hautement à la Sainte Vierge. Et personne n'avait parlé du vin de Cana à Capharnaüm, ni sur le marché, ni sur les quais. Les bourgeois peut-être.

(20) L'Antéchrist, suivi de Ecce homo, textes et variantes établis par Giorgio Colli et Mazzino Montirani, traduction par Jean-ClaudeHéméry,
Paris, Folio Essais, 1990 [1974], p. 17.

(21) Ibid., p. 19.

(22) Ibid., p. 26. 
Le miracle de Cana a peut-être eu lieu, mais son effet est quasiment nul sur un peuple pour qui le miracle est avant tout celui de la vie, qu'il exprime d'ailleurs, face aux imprécations monochromes du Christ contre sa mère, par une explosion de couleurs: la «salle à manger verte et rose» traduit le fond pulsionnel coloré de l'existence, la puissance colorante d'une vie qui ne se renie point. Le prestige des couleurs souligne la permanence de la vie malgré la présence, potentiellement sombre, du Christ. C'est la revanche des couleurs de la vie face aux prétentions mélancoliques du christianisme. D'ailleurs, tout est mouvement dans cette cité de l'affirmation qui balaie la négativité du christianisme: «les voitures fil[ent]». Le narrateur commente: il s'agit d'«un mouvement, assez fort pour ce bourg», comme si la quantité d'énergie miseen jeu débordait, excédait les limites de la Cité, réclamait une autre architecture en vue d'une circulation appropriée, maximale, des forces. Rimbaud donnera corps à ce projet architectural dans les deux poèmes en prose de Villes. «[T] devoir être trop content ce soir-là» écrit le poète. C'est une définition de la vie comme excès de joie: une manière de gai savoir. La situation urbaine est en parfaite harmonie avec les forces de la nature, pour une définition de la vie comme énergie.

\section{Un Jésus plus humain}

Le peuple galiléen tend donc à conserver la vie forte, quelles que soient les imprécations mélancoliques du christianisme. Le peuple galiléen - un peu de ce peuple nouveau dont rêve Rimbaud dans Matin - existe par opposition à l'idéal ascétique chrétien: à la vie triste s'oppose la vie forte, à la négation de la réalité son affirmation majestueuse. Pour autant, le Christ que nous présente Rimbaud est différent: il s'agit d'un Christ profondément humain, et en quelque sorte non chrétien. Dans l'étude qu'il a consacrée à ces proses, Jean-Luc Steinmetz estime que, dans la deuxième prose dite «évangélique», le Christ tente de se dégager des instances parentales ${ }^{23}$. Le Christ souhaite échapper à la sémiotique maternelle et se retirer de la symbolique paternelle afin de fonder son humanité. Mère et Père poussent Jésus à accomplir sa mission divine, mais l'on perçoit chez lui une manière d'énervement. Rimbaud le précise, qui écrit que le Christ «avait parlé un peu hautement à la Sainte Vierge»; face à l'officier royal - dont Jean-Luc Steinmetz précise qu'il véhicule potentiellement pour Jésus l'image du Père ${ }^{24}-$, son agacement est encore plus manifeste:

Jésus retira sa main: il eut un mouvement d'orgueil enfantin et féminin. «Vous autres, si vous ne voyez des miracles, vous ne croyez point».

Certes, le Christ ne rejette pas totalement sa mère et, en guérissant le fils de l'officier royal («Allez, votre fils se porte bien»), il accepte de réaliser les Écritures. S'il accepte donc l'origine divine qui est la sienne, ce n'est pas sans une certaine mauvaise humeur (un coup de mauvais sang?), comme s'il désirait autre chose. Le texte suggère l'existence d'un Jésus pleinement humain, sans père ni mère, sans famille, qui tente d'inscrire son action par delà les exigences paternelles et l'insistance maternelle. Le Christ retrouve en effet une voie vers le monde, tel le chemin que tente de se frayer le damné de la Saison à travers l'enfer des représentations factices. Ce que souhaite véritablement ce Jésus humain, c'est ce que lui interdit sa mission même, à savoir «continu[er] par les rues moins fréquentées» - Rimbaud le bohême sait bien de quoi il en retourne. C'est, en effet, un type formidable de distraction, de désengagement que noue la dernière phrase. Pierre Brunel a raison d'écrire que «[1]e jour, - comme plus 
haut la force de la jeunesse -, ce sont là les vraies divinités: et Jésus le sait bien ${ }^{25}$. Le renouvellement de l'énergie humaine passe par la reconnaissance de l'énergie propre au monde, par delà les signes, les devoirs, l'obligation qui est celle de Jésus de réaliser les Écritures. Le Christ fonde l'énergie humaine alors que telle n'était pas sa mission: la nouvelle orientation que le voyant donne à son œuvre et à sa vie se laisse deviner par delà la fiction évangélique. Jusqu'à présent, le Christ était effacé et se contentait d'une existence et d'une parole secondes, qui consistaient à réaliser ce qui avait été annoncé jadis par les prophètes. Mais, selon son désir secret, qui est celui du réel, le Christ jouit de l'explosion de l'Ouvert. Jésus connaît la révélation d'une autre manière de voir au-delà de l'enfer(mement) de sa mission divine: il voit enfin le petit, scrute l'épiphénomène. Drôle de conversion que celle du Christ au monde réel, au rythme du monde. Rimbaud ne cesse de s'inventer à travers le personnage du Christ. En effet, à la fin de cette deuxième prose, une des sorties possibles et même probables de l'enfer est énoncée: en la matière, il s'agit de rompre avec l'obligation d'inventions nouvelles, d'opposer la valeur de l'instant au Temps des Signes et de faire taire en soi les «nobles ambitions $\gg^{26}$ pour rejoindre et s'adjoindre la gloire naturelle mais insondée de ce monde-ci. La transformation la plus radicale passera peut-être par la reconnaissance de ce monde-ci, qui est révélé, dans la deuxième prose dite «évangélique», à l'occasion d'une bifurcation dans l'itinéraire fortement balisé du Christ. Le côté enfantin et féminin de Jésus le dispose par ailleurs à cette célébration de la présence. Rimbaud traque et intensifie les particularités du texte johannique, dont le Christ est bien plus humain (ailleurs «bête à miracles», «machine signifiante») que dans les Synoptiques $^{27}$. Rimbaud insiste sur la dimension fortement humaine du Christ johannique, dont l'Évangile commence par ces mots, comme je l'ai déjà indiqué: «Au commencement était le Verbe, et le Verbe était avec Dieu, et le Verbe était Dieu» ${ }^{28}$. Au verset 14, l'Évangéliste indique: «Et le Verbe a été fait chair, et il a habité parmi nous; et nous avons vu sa gloire, comme la gloire du Fils unique du Père, étant plein de grâce et de vérité ${ }^{29}$. Le texte évoque le mystère qui est celui de l'Incarnation. Pour Rimbaud, la Parole faite chair est avant tout chair et, de ce fait, Jésus semble subir l'«entraînement» propre à sa nature. De manière comparable, le voyant s'est égaré parmi «les rêves les plus tristes ${ }^{30}$, esclave des signes et victime de sa propre prétention. À ce niveau, adhérer au monde commun, le retrouver, à nouveau le fouler alors que le voyant comme le Christ ne se voulaient pas de ce monde-ci, est aujourd'hui pour eux deux (mais «tout un» en Rimbaud) la seule planche de salut, le seul moyen de sortir de l'enfer des représentations mentales. Le spectacle de la nature, qu'enfante une énergie libre et qui est en ce sens parfaitement humain, est le pharmakon dont le Christ et le voyant avaient besoin. Il n'est pas étonnant que le prosateur évoque, en ce spectacle même, deux plantes reconnues pour leur vertu de guérison: le liseron et la bourrache. Le Christ et le voyant n'étaient malades que des missions (impossibles et inhumaines) qui étaient les leurs. Tous deux cherchent désormais à fonder, par delà les programmes monstrueux et les trusts psychiques aberrants, l'énergie humaine, comme un prolongement de la puissance de la nature, mais désormais sans orgueil ni vanité, dans les valeurs et les faits du «simple».

(25) Une saison en enfer, édition critique par Pierre Brunel, Paris, José Corti, 1987, p. 125, n. 16. fer.

(26) Cette expression figure dans Nuit de l'en-

(27) Comme le souligne Paola Ricciuli, «[1]e Christ de saint Jean est un homme qui connaît la fatigue, comme quand il s'assied près du puits de Jacob, il connaît la douleur, la colère, l'amitié, faisant de la Foi le thème central de sa mission» (art. cit., p. 108).

(28) Evangile selon saint Jean, I,1.

(29) Évangile selon saint Jean, I, 14

(30) Cette expression figure dans Délires II-Alchimie du Verbe. 
Le statut de la parole

La deuxième prose dite «évangélique» est la seule où Jésus prenne la parole. Alors que dans la première prose il n'a rien pu dire et que dans la dernière il est étrangement taciturne, ci-présent il se manifeste en tant qu'une vérité faite parole. Le Christ est-il vraiment cette vérité de parole? Il est vrai que l'adaptation de Rimbaud est très proche de l'original, mais c'est l'énonciation évangélique qui intéresse au premier chef le poète. Rimbaud n'a pas choisi de faire parler le Christ à n'importe quel moment, mais à l'instant même où il consacre une certaine énergie de la parole, laquelle cesse de prendre l'allure d'un cryptogramme (le langage «parabolique») pour se faire violemment affective. Le langage cesse alors d'être le lieu où se dépose une vérité à révéler, il se lie avec l'humeur, devient rythme. Langage-rythme et non plus langage des signes. La parole christique participe paradoxalement de la poétique de l'énergie. La deuxième prose dite «évangélique» annonce donc ce livre de colère dans la violence langagière qu'est Une saison en enfer, alors que la première et la troisième de ces mêmes proses ont trait au fait de ne plus savoir parler. Ce retrait de la parole ne coïncide pas avec le silence. Rimbaud est donc moins sensible à cette parole de vérité, comme l'annonce le liminaire de l'Évangile selon saint Jean, qu'au fait que celle-ci puisse désormais être porteuse de colère (comme dans Une saison en enfer). La parole de Jésus ne se nourrit plus des injonctions du Père, mais forme le côté violemment humain, charnel, de Jésus. Elle est une parole restituée à son fond pulsionnel. Rimbaud indique que le Christ a parlé un peu hautement à la Sainte Vierge. C'est comme si le langage possédait en son tréfonds une réserve de colère, comme si s'y était déposée une vérité, mais cette fois de l'ordre de l'intensité. In vino veritas? En tout cas, le vin semble délier la langue. Rimbaud interprète comme une déclaration d'indépendance cette parole un peu haute adressée à la Sainte Vierge. Jadis suturée à l'Un, la parole se dégage pour exprimer désormais la vérité non plus de l'Un, mais du sujet. De même, lorsque Jésus dit: «Vous autres, si vous ne voyez des miracles, vous ne croyez point», ce propos s'affiche comme étonnamment précurseur de la tonalité d'Une saison en enfer. Il ne faut plus désormais réclamer des miracles à la parole, mais la laisser être ce qu'elle peut. L'idéal nouveau de la parole rimbaldienne, à l'orée d'Une saison en enfer, semble désormais contenu dans ce tour performatif et sans fioritures: «Allez, votre fils se porte bien».

\section{La troisième prose dite «Évangélique»}

\section{Dérives mélancoliques}

La troisième prose dite «évangélique ${ }^{31}$ est d'un intérêt extraordinaire et se présente comme fort proche de l'Adieu d'Une saison en enfer, au cours duquel Rimbaud finit par éloigner de lui le monde mortifiant de la négativité et se détacher de sa paradoxale passion pour l'énergie infernale. Ce poème en prose - puisque tel il doit être défini, étant d'une beauté comparable aux Illuminations - exprime un univers lourd d'une mélancolie véritable, dans ce qu'elle peut avoir de terrible. Rimbaud met en effet en scène cet ennui pour lui inséparable de l'énergie ${ }^{32}$. Chaque élément, composant mélancolique est disposé, proposé avec soin par le poète, lequel n'a d'autre but que

(31) Quelques idées à propos de cette dernière prose ont été développées sous le titre «L'envers de Bethsaïda», Parade sauvage, $\mathrm{n}^{\circ} 16$, mai 2000, p. $58-65$.

(32) Sur «l'énergie de l'ennui», voir Yves CHAR-
NET, Figurer l'énergie: Baudelaire écrivain du visuel, thèse de doctorat soutenue le 25 novembre 1995 à l'Université Charles de Gaulle-Lille III, volume 1, p. 2-80. 
de mettre sous les yeux du lecteur cette «phosphorescence de la pourriture» ${ }^{33}$ dont parle Baudelaire:

Bethsaïda, la piscine des cinq galeries, était un point d'ennui. Il me semblait que ce fût un sinistre lavoir, toujours accablé de la pluie et noir, et les mendiants s'agitant sur les marches intérieures, - blêmies par ces lueurs d'orages précurseurs des éclairs d'enfer, en plaisantant sur leurs yeux bleus aveugles, sur les linges blancs ou bleus dont s'entouraient leurs moignons.

D’une part, la piscine de Bethsaïda est considérée comme un «point d'ennui», comme une sorte de stase immense de la tristesse qui n'est pas sans rappeler ce «trou» qu'est Roche pour le poète impatient de mai 1873. La nature dépressive, en liquéfaction (la «pluie»), à laquelle répond une humanité navrante (les «mendiants»), concourt à créer un climat de désolation, de déréliction. D’autre part, à cette inertie répond une sorte d'activité. La mélancolie possède son énergie propre: «les mendiants s'agit[ent]», les marches intérieures sont traversées par «[d]es lueurs d'orages précurseurs des éclairs d'enfer». La prose est ainsi chargée d'une électricité énorme, d'une énergie qui investit, jusqu'à l'embraser, ce «point d'ennui» qu'est la piscine. Le lien de la mélancolie et de l'énergie, leur secrète mise en regard, débouche sur ce que Baudelaire nomme «quelque chose d'ardent et de triste» ${ }^{34}$, ou encore cette «luxuriance du deuil » ${ }^{35}$ qu'évoque Antoine Raybaud à propos de Guerre et d'Enfance. Les mendiants eux-mêmes sont des êtres animés d'une énergie paradoxale, produits d'une hybridation réussie de la faiblesse et de la force; aux diverses infirmités des Damnés s'ajoutent les couleurs flamboyantes du blanc et du bleu, lesquelles confèrent à ces hommes un bizarre rayonnement; il s'agit bien, comme l'explique Baudelaire, de rendre phosphorescente la «pourriture», de créer de nouveaux mixtes à même d'exprimer l'intensité propre à la mélancolie. Carences et couleurs se mêlent, se répondent et constituent ces nouveaux agencements mélancoliques dont Baudelaire et Rimbaud entreprennent la folle et vaine exploration. Le caractère terrifiant de l'évocation s'en trouve renforcé, puisqu'un feu éclaire enfin l'abîme de noirceur de la mélancolie et en donne à voir l'horreur, ouvrant d'une certaine manière celle-ci au représentable. L'intensification ouvre le donné mélancolique à l'espace du visible, comme si, en leur nuit même, les corps souffrants des mendiants avaient trouvé leur lumière intérieure. Mieux qu'à l'hypotypose, Rimbaud se fie au pouvoir de l'«energeia» pour réactiver le texte évangélique, lui redonner vie ${ }^{36}$. Plus que jamais, Rimbaud «énergétise» sa poésie et témoigne d'une sensibilité extrême - celle peut-être du Christ qu'il présente, dans la deuxième prose dite «évangélique», comme un être «enfantin et féminin». Le poète extrait de toute chose, de chaque être, des puissances de vie insoupçonnées. Il réveille le corps des choses mortes: il est celui qui confère à l'Évangile sa poésie, qui donne à l'ennui son énergie. Jean-Luc Steinmetz a ainsi raison d'écrire que la «lumière à la fois infernale et mystique [...] semble [...] la principale détentrice de la scène ${ }^{37}$. À travers cette évocation l'on perçoit à quel point Rimbaud est proche des idées du XVIII ${ }^{\mathrm{e}}$ siècle; à l'instar de Diderot, il a foi en «l'énergie de nature»

(33) Euvres complètes, tome II, éd. cit., p. 317318.

(34) Ibid., p. 72

(35) Fabriques d' "Illuminations", Éditions du Seuil, 1989, pp. 187-201.

(36) Comme le souligne Claude Calame, «[l']enargeia est donc le mode privilégié de la manifestation sensible, l'effet de la connaissance empirique immédiate, le critère de la vérité objective, essentiellement par le moyen de la vue» (cité par Yves LE BOzEc, L'bypotypose: un essai de définition formelle,
«L'information grammaticale», $\mathrm{n}^{\circ}$ 92, janvier 2002, p. 4). De fait, c'est «[l']enargeia qui pose le concept phare de l'hypotypose» (ibid., p. 4). L'énergie n'est toutefois pas réductible à la seule figure de l'hypotypose, qui n'en constitue que l'une des formes d'expression. Il est à noter que Rimbaud n'ignorait rien de ce procédé, comme le montrent, dans l'Album zutique, les Hypotyposes saturniennes ex Belmontet.

(37) Éd. cit., p. 137. 
comme en «l'énergie humaine» ${ }^{38}$. Toujours il se révèle sensible à «[1]a circulation des sèves inouïes», à «l'éveil jaune et bleu des phosphores chanteurs [...]», comme l'écrit le poète dans Le Bateau ivre. Le premier paragraphe de la troisième prose dite «évangélique» est un chant à la gloire de la mélancolie ardente, avec en regard le texte de saint Jean, à la fois comme canevas à traiter énergiquement et comme apparat sans rival, en vue d'une célébration particulièrement réussie, même s'il s'agit précisément de chanter le désastre.

\section{Le Christ... en amateur de singularités}

À l'heure de la Saison, que peut bien représenter l'expérience christique pour Rimbaud? Le début de la troisième prose dite «évangélique» n'est certes pas sans évoquer l'entrée en matière d'Adieu, ni plus largement ce fort singulier climat tout à la fois d'énergie et de tristesse qui caractérise dans sa globalité Une saison en enfer. Alain Coelho fait à juste titre remarquer que «[1]es Proses évangéliques [...] sont transparentes sur ce point. La perspective n'est pas celle de la croyance ou non en Dieu, mais celle de l'intériorisation par Rimbaud de la trajectoire, de la figure et de la sensibilité probable du Christ» ${ }^{39}$. Cette fameuse sensibilité est une disposition à accueillir chez le Christ toutes les singularités de l'existence, les énergies particulières. Le Christ participe d'un univers où règne l'anomie, et bien que sa Parole se veuille souveraine et liée à l'Un (et de fait soudée à la Représentation, à la catégorie de l'Identité $^{40}$ ), il ne cesse de vivre aux marges, à la bordure, et n'en connaît que mieux dès lors les puissances de décentrement, les forces de finitude. Tel est en effet le mode d'existence choisi par le Christ, perdu parmi les naufragés, les rebuts de l'humanité, partageant à dessein le destin de ces êtres anomiques dont Rimbaud sait bien que se dégage une secrète félicité. Le lavoir est ce lieu intensif où se déploie une mélancolie pure. Jésus est celui qui consomme jusqu'à la lie la coupe de négativité de l'existence mortelle, qui engrange les délires successifs de chacun, qui est l'ami pour les «incon-

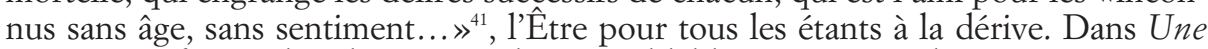
saison en enfer, Rimbaud témoigne d'une semblable passion pour l'anomie et, comme Jésus, il dresse une carte des singularités en présence. En la matière, les Évangiles sont peuplés d'infirmes, de lépreux, d'aveugles, d'autant de singularités sauvages. Pour le Christ, «il s'agi[t] de prolonger une singularité jusqu'au voisinage d'une autre, de manière à produire une "configuration d'événements", c'est-à-dire l'ensemble le plus riche ou le plus consistant possible $»^{42}$. C'est une «connexion, [une] conjonction de singularités ${ }^{43}$ au cours de laquelle le Christ se livre à une «[e]xploration de voisinages $\gg^{44}$. A cet effet, le christianisme se présente comme une religion passablement «tordue» - et le propos de Rimbaud est certes, sur ce point, différent de celui de Nietzsche dans L'Antéchrist. Dans les Évangiles, Jésus émet clairement l'idée qu'il est là pour les péchés, pour connaître cette énergie propre à la négativité sur laquelle Rimbaud a fondé la majeure partie de son œuvre. Pour Rimbaud, le Christ est celui qui est hanté par le $\mathrm{Mal}$, affronte tout le négatif de la vie, habite parmi les êtres souffrants. Si le christianisme est le garant de l'histoire officielle, s'il a mis la Représenta-

(38) À ce sujet, voir Jacques Choulllet, Diderot, poète de l'énergie, Paris, Presses Universitaires de France, «Écrivains», 1980, p. 11-26, et MiCHEL Delon, L'idée d'énergie au tournant des Lumières, Presses Universitaires de France («Littératures modernes»), 1988, p. 243-279.

(39) Une saison en enfer: fin de la littérature, préface de Jean-Luc Steinmetz, Joseph K. («Essais»), 1995 , p. 186.
(40) Sur cette question, voir JEAN-LuC STEINMETZ, éd. cit., p. 141.

(41) Cette expression figure dans Adieu.

(42) Gilles Deleuze, Périclès et Verdi: la philosophie de François Châtelet, Paris, Les Éditions de Minuit («Critique»), 1988, p. 19.

(43) Ibid., p. 23.

(44) Ibid. 
tion en lieu et place du réel, en revanche le Christ en est la bordure, la limite, l'impensé. Le Christ est partout où de l'énergie négative se produit, là où les anomalies se déploient. Même s'il ne fait que réaliser l'Écriture des Prophètes ${ }^{45}$, le Christ entraîne dans un devenir-intensif le monde de la Loi et se retrouve inévitablement confronté à l'énergie humaine. Dans la deuxième prose dite «évangélique», l'on est même en droit d'estimer qu'il est «débauché» par l'énergie du monde créé:

Jésus dit: «Allez, votre fils se porte bien». L'officier s'en alla, comme on porte quelque pharmacie légère, et Jésus continua par les rues moins fréquentées. Des liserons, des bourraches montraient leur lueur magique entre les pavés. Enfin il vit au loin la prairie poussiéreuse, et les boutons d'or et les marguerites demandant grâce au jour.

Pris dans la danse rythmique de la nature, le Christ semble un instant se libérer de l'emprise qu'ont sur lui les signes d'une transcendance vide ${ }^{46}$. C'est bien le mystère de l'Incarnation qui captive Rimbaud. Le Christ serait-il malade des intensités, un voyant (voyeur?) se repaissant des pêchés, en tirant même jouissance? Or c'est précisément ce Christ-là que nous présente Rimbaud dans la troisième prose dite «évangélique».

\section{Le problème des «agencements»}

Le deuxième paragraphe participe d'une autre perspective et constitue une tentative de contestation, voire de renversement des valeurs consacrées par le début de la prose. Bien que soit annoncée comme imminente la venue de Jésus (puisque «[c']est là qu'[il] fit [1]a première action grave»), la préséance revient encore et toujours à l'énergie et aux agencements de type positif ou de type négatif adjacents:

C'est là que Jésus fit la première action grave; avec les infâmes infirmes. Il y avait un jour, de février, mars ou avril, où le soleil de $2 \mathrm{~h}$. ap. midi, laissait s'étaler une grande faux de lumière sur l'eau ensevelie; et comme, là-bas, loin derrière les infirmes, j'aurais pu voir tout ce que ce rayon seul éveillait de bourgeons et de cristaux et de vers, dans ce reflet, pareil à un ange blanc couché sur le côté, tous les reflets infiniment pâles remuaient.

Pour Jean-Pierre Giusto, qui analyse l'imagination matérielle du poète, «Bethsaïda nous présente le tableau de la métamorphose de l'eau sombre en eau claire sous l'influence du soleil $\gg^{47}$. Le critique n'hésite pas à attribuer la responsabilité de cet éveil au poète lui-même et le place parmi «les succès du rêveur»: «Rimbaud, fils du soleil, est l'ange-rayon qui vient métamorphoser l'eau stagnante de la piscine de Bethsaïda $\gg^{48}$. Il est vrai que dans la noirceur de l'évocation biblique vient brutalement s'inscrire, à un moment où on ne l'attend guère, le «je» du narrateur: cette incursion, nullement innocente, a pour effet de soumettre à la question le lien séculaire qui existe entre l'énergie et la négativité. À ce moment précis, le narrateur semble accompagner l'action du soleil et tenter de conjurer la dimension destructrice de l'énergie, en la réinscrivant in medias res dans le circuit étroit de la positivité. Il émet l'hypothèse suivant laquelle un agencement de l'énergie «de type positif» (intensité-positivité)

(45) Voir Jean-Luc Steinmetz, op. cit., p. 132.

(46) Après «sûre», le manuscrit indique l'existence d'une phrase partiellement biffée: «Un signe de vous, ô volonté divine; et toute obéissance est prévue presque avant vos». D’une interprétation difficile, cette leçon du manuscrit (d'une lecture conjecturale également) montre que l'extrême obéissance de Jésus à sa mission divine constitue en fait pour lui un problème, provoque une souffrance chez un être nécessairement déchiré entre son devoir divin et la conscience humaine qui l'habite. Entre le monde des signes et celui des rythmes, le Christ ne peut choisir.

(47) Rimbaud créateur, Paris, Presses Universitaire de France, 1980, p. 38.

(48) Ibid., p. 261. 
pourrait prendre la place des agencements «de type négatif» (intensité-négativité: la mélancolie «intense» du premier paragraphe). Le narrateur évoque ce «rayon seul», songe à la pureté primitive d'une énergie qui ne serait pas entrée dans un désastre combinatoire avec le Malheur. Rimbaud réfléchit à un changement d'agencement, rêve d'une énergie passant outre les obligations mélancoliques, le détournant ainsi des «marécages de sang», des «abîmes de boue» de la modernité maudite ${ }^{49}$. C'est pourquoi le poète prolonge l'action du soleil. Bien que l'énergie soit en majeure partie responsable du destin funeste du poète, ce dernier sait bien qu'elle pourrait être également sa planche de salut, à la condition impérative de changer d'agencement, d'inverser les combinatoires; que, désormais, cette énergie, loin de transformer les hommes en «monstres», leur permette de séjourner de nouveau dans la pure nature et purge conséquemment la création poétique de sa négativité (Rimbaud évoque à dessein les «vers »). Ce vœu n'est pas sans rappeler celui formulé par Baudelaire dans La Muse malade:

Je voudrais qu'exhalant l'odeur de la santé

Ton sein de pensers forts fût toujours fréquenté,

Et que ton sang chrétien coulât à flots rythmiques,

Comme les sons nombreux des syllabes antiques

Où règnent tout à tour le père des chansons,

Phœbus, et le grand Pan, le seigneur des moissons ${ }^{50}$.

Une même dialectique de la négativité et de la positivité de l'énergie est à l'œuvre dans Enfance, poème d'une insistante mélancolie que le poète tente de conjurer par le biais d'une permutation des agencements. À une telle «luxuriance du deuil» doit se substituer quelque énergie positive, ce que la prose de Bethsaïda semble bien réaliser, mais sans pour autant que la dimension invariablement inquiétante des intensités soit abolie. Il n'est donc pas si simple de changer d'agencement. «Bethsaïda [...]» convie en fait à un misérable miracle. La suite de la prose l'indique clairement: «Les premiers entrés sortaient guéris, disait-on. Non». Cet extrait n'indique pas obligatoirement que le miracle n'a pas eu lieu, mais plutôt que sa force bénéfique n'est pas suffisante pour vaincre la négativité ambiante. Un indice présent dans le deuxième paragraphe permet d'ailleurs de conclure en ce sens sans qu'il faille se reporter pour cela à la suite de la prose. Le rayon ne change pas vraiment «l'eau sombre» en «eau claire»; inévitablement, des traces de négativité subsistent, puisque les «reflets» restent «infiniment pâles». La pâleur est presque l'absence de couleur. Infinie, elle est l'abîme de l'absence de couleur. La négativité fait donc un retour en force après le bref laps de temps alloué au rêve. L'heure reste celle du règne des agencements de l'énergie de type négatif. Toutefois il s'agit peut-être déjà d'une sorte d'entrevision d'une possible sortie de l'enfer, même si la puissance propre à la négativité demeure considérable.

\section{L'énergie du mal}

Indépendamment des personnages ou des sujets qui, assurément, ne sont que des déterminations secondaires, il existe une certaine «énergie du mal» (pour reprendre la fameuse expression de Baudelaire à propos des Liaisons dangereuses $\left.{ }^{51}\right):$ «[...

(49) BAUdELAIRE, «Réflexions sur quelques-uns de mes contemporains», in Euvres complètes, tome II, éd. cit., p. 168.

(50) Les Fleurs du Mal, édition établie par Jean
Delabroy, Magnard («Texte et contextes»), 1989, p. 66.

(51) «Notes sur les Liaisons dangereuses», in Euvres complètes, tome II, éd.cit., p. 68. 
tous les péchés, fils légers et tenaces du démon, qui pour les cœurs un peu sensibles, rendaient ces hommes plus effrayants que les monstres, voulaient se jeter à cette eau». Rimbaud insiste sur le fait que ce n'est que dans un second temps ou sur un second plan que l'énergie est rapportée à une forme-sujet (les «hommes»). Dans la constitution d'un personnage, on peut distinguer deux plans distincts : «plan de consistance ou de composition des heccéités dans un cas, qui ne connait que des vitesses et des affects, - tout autre plan des formes, des substances et des sujets dans l'autre cas $\gg^{52}$. Dans la poétique rimbaldienne, le premier plan est largement prioritaire; c'est l'énergie du mal qui rend effrayants ces hommes. Il ne s'agit donc pas d'écrire qu' «être effrayant» est un défaut, ni même une caractéristique de type psychologique ou physiologique. «L'effrayant» existe en tant que tel. Il est déterminé par un certain degré dans l'intensité, qui peut être éventuellement rapporté à un sujet: «hommes plus effroyables que les monstres». «Ma blessure existait avant moi, je suis né pour l'incarner» écrit Joë Bousquet ${ }^{53}$. L'énergie du mal existe, mais seule. Elle prend la forme de péchés, «fils légers et tenaces du démon». Elle se caractérise donc par une existence mobile («légers») et en même temps profondément matérielle («tenaces»). Les hommes ne sont que les «corps-hôte[s] $\gg^{54}$ de cette énergie autonome: la modification de la physionomie de ces «hommes» est le signe d'une possession. L'énergie les a totalement investis.

\section{Puissance de la négativité}

L'autonomie qui caractérise la négativité garantit sa force. Le narrateur peut alors éprouver la capacité de résistance qui est celle de la négativité en dépit de la promesse de guérison qui a été faite: «Les premiers entrés sortaient guéris, disait-on. Non». Le remède de lumière s'avère donc incapable de contrebalancer les forces réactives. De ce fait, les corps sont violemment rejetés sur les marches: le rapport de forces s'établit au bénéfice de la négativité. La négativité n’est pas si aisément congédiable. Rimbaud en fera l'amère expérience dans Une saison en enfer, où tant de lignes de fuite sont colmatées avant que la «victoire» ne soit (définitivement?) «acquise».

\section{Le pharmakon}

On perçoit bien ce qui peut rapprocher Rimbaud de la figure du Christ. Le Christ vit parmi les êtres anomiques, singuliers, à l'instar du poète qui, dans Délires $I$, prend place parmi le «bétail de la misère». Si le poète a pour la négativité un intérêt fondamental (qui se changera en souffrance à l'occasion d'Une saison en enfer), de son côté le Christ y est continuellement confronté. Il est à sa manière un voyant puisque, dans la prose de Bethsaïda, il visionne littéralement de l'intérieur tous ces êtres dont il s'est fait l'obscure compagnie: «Le divin maître se tenait contre une colonne: il regardait les fils du Péché; le démon tirait sa langue en leur langue; et riait ou niait». Par la suite, le seul regard du Christ suffit-il à guérir le Paralytique? En tout cas, le mouvement final de la prose est analogue à celui qui commande Adieu au cours duquel le narrateur forme le projet d'entrer aux «splendides villes». Est-ce à dire que Rimbaud a foi dans le Christ pour l'extraire du monde de la négativité? Certes non, et c'est pour cela que, contrairement à ce qu'indique le texte johannique, le Christ ne

(52) Gilles Deleuze et Félix Guattari, Mille plateaux, Les Éditions de Minuit, coll. Critique, 1980 , p. 320.

(53) Phrase particulièrement célèbre, extraite des Capitales et souvent citée par Gilles Deleuze, notamment dans son dernier article: L'immanence, une vie..., Philosophie, $\mathrm{n}^{\circ}$ 47, septembre 1995, $\mathrm{p}$. 7.

(54) Pour reprendre l'expression employée par YoshiKazu NaKaji dans Combat spirituel ou immense dérision? Essai d'analyse textuelle d'«Une saison en enfer», José Corti, 1987, p. 53. 
profère aucune parole suite au «miracle». Ce qui intéresse au premier chef Rimbaud n'est autre que le pharmakon ${ }^{55}$, ce qui porte remède et permet d'envisager une sortie de l'enfer (les mendiants sont finalement considérés comme des «Damnés»). Nulle conversion, donc. L'essentiel est en effet qu'un prisonnier de l'enfer ait pu s'échapper. Et c'est peut-être Cecil Arthur Hackett qui a raison lorsqu'il écrit que le Paralytique s'en sort par ses propres moyens ${ }^{56}$. Le message final adressé par le narrateur à Jésus est en tout cas fort étrange, car si le Paralytique, qui figure évidemment d'avance le damné d'Une saison en enfer, finit par s'en aller, le Christ, lui, reste... en enfer.

La prose de Bethsaïda: «musicienne du silence»

Utiliser l'Évangile de la parole et le transformer en celui du silence du Christ constitue un véritable tour de force de la part de Rimbaud. Dans cette dernière prose, il est assurément moins question du fait que «Jésus n'a rien pu dire» à Bethsaïda que d'un absentement de la parole. Rimbaud conteste le pouvoir, et même la légitimité, de la parole christique. Le Christ est le témoin retiré qui assiste, impuissant, à la naissance d'un nouvel ordre: celui du démon, convulsif et volubile à la fois: «le démon tirait sa langue en leur langue; et riait ou niait». La troisième prose est ce moment de l'œuvre de Rimbaud où se referme la parenthèse évangélique pour un enfer à explorer et à vivre. Les proses dites «évangéliques» sont vouées à l'enfer, donnent une vue sur l'enfer... à écrire. Et à dire. Au risque du silence.

YANN FRÉMY

(55) Le thème du pharmakon apparaît déjà à la fin de la deuxième prose dite «évangélique». Comme le rappelle Pierre Brunel dans son édition critique d'Une saison en enfer (p.140, n.42), «[1]a bourrache est un diurétique et un diaphorétique, le liseron un purgatif (liseron scammonée d'Asie Mineure) 》.

(56) Voir Arthur Rimbaud, Euvres poétiques, textes présentés et commentés par C.A. Hackett, 